\title{
Correction to: Evaluation of collateral circulation based on a single time-variant color map of multi-phase CTA and its association with CTP parameters
}

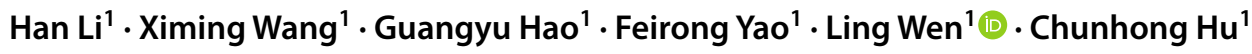

Published online: 25 August 2021

(c) Springer Nature Singapore Pte Ltd. 2021

Correction to: Chinese Journal of Academic Radiology (2021)
https://doi.org/10.1007/s42058-021-00069-z

The original version of this article, published on May 18, 2021, contained a mistake.

The first and last names were interchanged and the spelling of Ling Wen's name was incorrect. The original article has been corrected.

The original article can be found online at https://doi.org/10.1007/ s42058-021-00069-z.

\section{Ximing Wang}

wangximing1998@163.com

$\bowtie$ Ling Wen

wenling@suda.edu.cn

1 Department of Radiology, Institute of Medical Imaging, The First Affiliated Hospital of Soochow University, Soochow University, No. 899 Pinghai Road, Suzhou 215006, Jiangsu, China 